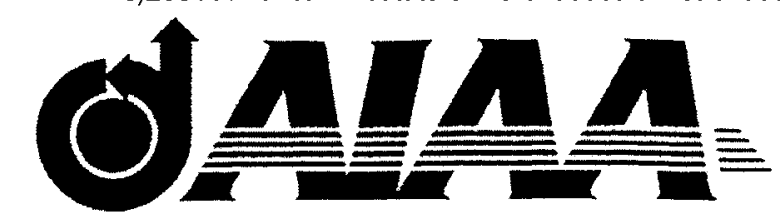

\title{
AIAA 2001-0698
}

\section{CINEMAGRAPHIC PIV INVESTIGATION OF THE LIFTED TURBULENT JET DIFFUSION FLAME STABILIZATION REGION}

Ansis Upatnieks*, Steven L. Ceccio ${ }^{\dagger}$, James F. Driscoll ${ }^{*}$

*Department of Aerospace Engineering ${ }^{\dagger}$ Department of Mechanical Engineering

University of Michigan Ann Arbor, MI 48109-2140

\section{$39^{\text {th }}$ AIAA Aerospace Sciences Meeting \& Exhibit 8-11 January 2001 / Reno, NV}


AIAA-2001-0698

\title{
Cinemagraphic PIV Investigation of the Lifted Turbulent Jet Diffusion Flame Stabilization Region
}

\author{
Ansis Upatnieks*, Steven L. Ceccio ${ }^{\dagger}$, and James F. Driscoll ${ }^{\dagger}$ \\ University of Michigan \\ Ann Arbor, Michigan 48109-2140
}

\begin{abstract}
The time evolution of the the velocity field and flame thermal boundary at the stabilization region of a lifted turbulent jet diffusion flame are observed using a high-speed Cinemagraphic Particle Image Velocimetry (CPIV) system. CPIV images are obtained at high acquisition rates ( $8000 \mathrm{images} / \mathrm{s})$ and long sequence lengths (1100 images) compared to the characteristic time scales of the flow, showing relatively long histories of time incremental changes in the velocity field and the flame boundary. The velocity of the flame stabilization point and the gas velocity are determined simultaneously, providing a measurement of the flame propagation velocity. The measured average gas velocity $\left(0.8 \mathrm{~S}_{\mathrm{L}}\right)$ and flame propagation velocity $\left(0.2 \mathrm{~S}_{\mathrm{L}}\right)$ at the stabilization point are lower than the laminar flame speed $\mathrm{S}_{\mathrm{L}}$. Intermittent flow reversal is observed in isolated regions immediately upstream of the stabilization point. The flow reversal is correlated with upstream flame motion, inward radial gas velocities, and eddy passage just inward of the stabilization point. The flow reversal may be induced by thermal expansion associated with the combustion process. This mechanism could play a significant role in turbulent jet flame stabilization because the induced velocities are similar to the measured flame propagation velocities.
\end{abstract}

\section{Nomenclature ${ }^{\S}$}

$\mathrm{V}_{\mathrm{p}} \quad=3-\mathrm{D}$ flame propagation velocity

$\mathrm{V}_{\mathrm{f}} \quad=3-\mathrm{D}$ flame boundary velocity

$\mathrm{V}_{\mathrm{g}} \quad=3-\mathrm{D}$ gas velocity

$\mathrm{n} \quad=$ Unit normal to flame

$U_{p} \quad=$ Normal component of $V_{p}$

$\mathrm{U}_{\mathrm{f}} \quad=$ Normal component of $\mathrm{V}_{\mathrm{f}}$

$\mathrm{U}_{\mathrm{g}} \quad=$ Normal component of $\mathrm{V}_{\mathrm{g}}$

$\mathrm{S}_{\mathrm{L}} \quad=$ Laminar flame speed

$\mathrm{S}_{\mathrm{T}} \quad=$ Turbulent flame speed

${ }^{\S}$ Variables are defined at the flame stabilization point.

\section{Introduction}

The lifted turbulent jet diffusion flame stabilization mechanism is one of the long-standing unsolved problems in combustion science ${ }^{l}$, in large part due to the difficulties in performing meaningful measurements in a turbulent, reacting flow field. For a certain jet velocity, the flame base stabilizes at some downstream

*Ph.D. Candidate. Member AIAA.

${ }^{\dagger}$ Professor, Mechanical Engineering.

${ }^{\ddagger}$ Professor, Aerospace Engineering. Associate Fellow AIAA.

Copyright (C) 2001 by Ansis Upatnieks. Published by the American Institute of Aeronautics and Astronautics, Inc. with permission. distance from the jet exit. The position of the base as well as all other flow characteristics are statistically stationary, but the instantaneous base position and gas velocity fluctuate continuously due to the interaction between the unsteady turbulent flow field and the combustion process. It has been reasoned that stabilization occurs where the flame base propagates with a sufficient velocity against the oncoming gas flow to maintain its average position ${ }^{2}$. The flame propagation velocity $V_{p}$ is defined as:

$$
V_{p}=\left(V_{f} \bullet n\right)-\left(V_{g} \bullet n\right)
$$

where $V_{f}$ is the velocity of the reaction zone, $V_{g}$ is the gas velocity, and $n$ is the normal to the reaction zone surface at the stabilization point where these velocities are measured. The normal $\mathrm{n}$ is always parallel to the jet axis. Determination of the instantaneous value of $V_{p}$ requires simultaneous knowledge of $V_{f}$ and $V_{g}$ at the stabilization point. Unfortunately, simultaneous determination of these quantities presents great difficulties for experimental measurement or numerical simulation because of the challenging nature of this turbulent, unsteady, reacting flow. The flame stabilization point position, $\mathrm{V}_{\mathrm{f}}$, and $\mathrm{V}_{\mathrm{g}}$ all fluctuate simultaneously and with relatively high characteristic frequencies. Single-point measurement techniques such as LDV can provide time-resolved measurements of the gas velocity but are restricted to fixed measurement 
points that cannot follow the rapidly fluctuating position of the flame stabilization point. Furthermore, it is not possible to determine the instantaneous value of $V_{f}$ from fixed single-point measurements. Fixed singlepoint measurements of the gas velocity $\mathrm{V}_{\mathrm{g}}$ at the average position of the flame base have yielded values significantly higher than the laminar flame speed $\mathrm{S}_{\mathrm{L}}{ }^{3}$, suggesting that the flame propagates with a higher turbulent flame speed $S_{\mathrm{T}}$ that exceeds $S_{\mathrm{L}}{ }^{2}$. However, measurements of $\mathrm{V}_{\mathrm{g}}$ at the instantaneous stabilization point have yielded values lower than the fixed singlepoint measurements ${ }^{4}$.

Instantaneous 2-D measurement techniques such as $\mathrm{PIV}$ and $\mathrm{PLIF}^{4,5}$ (Planar Laser Induced Fluorescence) can be used to determine the in-plane components of $V_{g}$ at the flame stabilization point. However, conventional single-shot PIV and PLIF do not yield any component of $V_{p}$ or $V_{f}$ because at least two successive observations of the stabilization point position are required to determine $V_{f}$ and hence $V_{p}$. Two-shot PIV/PLIF ${ }^{6-9}$ can provide simultaneous measurements of $V_{g}$ and $V_{f}$, but requires foreknowledge of $V_{f}$ and its time scales for choice of a suitable two-shot time delay. Too short of a time delay leads to errors as uncertainties overwhelm the measured velocities, while too long of a time delay does not capture high-frequency motions. Furthermore, conventional 2-D PIV and PLIF imaging techniques acquire images at insufficient rates compared to the length and time scales of these flows to observe the time evolution of the velocity field or the flame reaction zone.

Realistic numerical simulations of turbulent jet flame stabilization are not currently available. Reynolds numbers above 2300 are associated with lifted turbulent jet diffusion flames, and these conditions are beyond the computational capability of today's computers using Direct Numerical Simulation. Large Eddy Simulations are currently in an early stage of development and not yet used in a predictive sense.

High-speed CPIV provides a sufficient combination of spatial and temporal resolution for simultaneous, time-resolved observations of the velocity field and the flame position, enabling direct measurement of the inplane components of $V_{p}$. Up to 8000 instantaneous CPIV images per second are obtained, with sequence lengths exceeding 4000 images. The combination of high sampling rate and long sequence length allows identification of trends in the data, minimizing the influence of random uncertainties of individual data points. Low gas velocities can be measured as accurately as high velocities for the same sequence by adaptively cross-correlating images with the appropriate time separation. The time evolution of 2-D turbulent vortex structures and their interaction with the reaction zone can be observed

\section{Experimental Method}

\section{A. Test Flow Apparatus}

The test flow was a lifted turbulent jet flame with a low speed laminar air coflow. The jet consisted of methane ( $77 \%$ by volume) and nitrogen $(23 \%)$, and the jet Reynolds number was 4200 . The jet exit diameter and velocity were $5.0 \mathrm{~mm}$ and $14.0 \mathrm{~m} / \mathrm{s}$. The coflow outer diameter and velocity were $170 \mathrm{~mm}$ and 0.265 $\mathrm{m} / \mathrm{s}$. The flow was seeded with $3.5 \mu \mathrm{m}$ mean diameter $\mathrm{Al}_{2} \mathrm{O}_{3} / \mathrm{SiO}_{2}$ ceramic alloy particles (3M Zeeospheres, Type X-61). Seed particles were entrained into the flow with swirl-type seeders. Fig. 1 shows a schematic of the lifted flame apparatus.

\section{B. Image Acquisition}

Seed particles were illuminated by a pair of ClarkMXR ORC-1000 frequency doubled Nd:YAG lasers producing $300 \mathrm{~ns}$ duration, $6 \mathrm{~mJ}$ energy Q-switched pulses at a $4 \mathrm{kHz}$ repetition rate. The beam paths of both lasers were aligned using a beam combiner, expanded into a vertical sheet by a $80 \mathrm{~mm}$ focal length negative cylindrical lens, and focused to a $1.5 \mathrm{~mm}$ thickness at the camera imaging area by a $400 \mathrm{~mm}$ focal length biconvex lens. Fig. 2 shows a schematic of the Cinema-PIV (CPIV) diagnostics.

8000 CPIV images per second were acquired using a Photec $16 \mathrm{~mm}$ rotating prism movie camera. The sequence length exceeded 4000 images and the time delay between successive images was $125 \mu \mathrm{s}$, for a time record length exceeding $0.5 \mathrm{~s}$. The field of view was 32 $\mathrm{mm}$ (lateral) $\times 51 \mathrm{~mm}$ (axial) centered $80 \mathrm{~mm}$ downstream from the jet exit and $17 \mathrm{~mm}$ laterally from the jet axis. The CPIV image plane intersected only one side of the nearly axisymmetric flame base. The camera lens was an $80 \mathrm{~mm}$ Mamiya-Sekor macro (f8.0). The film type was Kodak double-X negative. Laser pulses were synchronized to the camera frame rate using Stanford Research Systems DG535 digital delay generators. TTL level frame rate output signals from the camera triggered the delay generators, which in turn triggered the laser Q-switches, producing pulses with the necessary delay and phase relationships for synchronization with the camera rotating prism.

In the two-frame cross-correlation PIV method employed, each film frame is illuminated by a single laser pulse, capturing particle positions at a particular instant. Separate frames may then be cross-correlated to obtain velocity data. Note that any two film frames can be cross-correlated, meaning that the same sequence can be analyzed with time delays from the minimum frame-to-frame period up to any multiple of this period. By adaptively selecting the crosscorrelation time delay, low velocities can be measured as accurately as high velocities for the same image sequence. This capability is particularly useful in flows 
with a high dynamic velocity range. In contrast, the fixed time delay of single-shot PIV yields much lower accuracy at low velocities than at high velocities.

An inherent operational feature of the rotating prism type camera employed is that significant variations occur in positioning of images onto the film (for purposes of two-frame cross correlation PIV). Therefore, the film perforations are not sufficiently accurate reference locations, and some other reference must be used. Placing fixed physical objects in the camera imaging plane to be imaged onto the film could provide the needed location reference, but would unacceptably influence the flow field. Instead, registration marks consisting of two pairs of crossed lines are projected into the camera field of view and imaged onto the film by the camera lens. No flow disturbance is created by the projection devices because they reside outside the flow path. Each pair of crossed lines is projected by a separate device with a backlighted chromed reticle pattern focused at the camera imaging plane using a $75 \mathrm{~mm}$ effective focal length lens assembly. Lighting for the projectors is taken from the $\mathrm{Nd}$ :YAG beams after they pass through the test section. A small portion of this residual light enters the single end of a dual-branch fiber-optic light guide and is channeled to both projectors. Ground glass diffuses laser light transmitted by the light guides to create even backlighting for the reticle patterns.

\section{Film Digitization}

The $16 \mathrm{~mm}$ film images were digitized using a Kodak DCS460 2036 × 3060 resolution digital color camera with a $52 \mathrm{~mm}$ Vivitar macro lens at f5.6 and Nikon PB6 bellows. The film was back-illuminated by a white light source with an opal glass diffuser. A desktop computer controlled the camera and a specially built motorized film-advance mechanism to automatically digitize the film. The RGB images were converted to 8 -bit grayscale. The digitized PIV images were aligned to a common reference using the crossed line registration marks made by the reticle projection devices. An analysis routine programmed in MATLAB located the coordinates of the intersections of the two crossed line pairs on each image and used standard image processing algorithms to align the images.

\section{Flame Thermal Boundary Measurement}

The flame thermal boundary was identified from the CPIV images by the distinct difference in seeding density across the boundary. The ceramic seed particles survive the flame, but their volume density decreases drastically along with the gas thermal expansion accompanying the combustion process. A MATLAB routine counted the number of seed particles within $128 \times 128$ pixel interrogation regions with $75 \%$ overlap, yielding a $53 \times 85$ grid of seeding density within the field of view. An automated routine found the isoline of seeding density that is half the average seeding density and located the furthest upstream point of the isoline as the stabilization point position.

\section{E. CPIV Data Reduction}

Two-frame cross-correlation analysis was performed on image pairs using AEA Visiflow PIV analysis software. The CPIV analysis was performed on the same grid and with the same interrogation regions and overlap as the thermal boundary analysis. Adjacent images with the minimum $125 \mu$ s time delay were cross-correlated to obtain all velocities up to the maximum within the field of view. Greater than 95\% valid vectors were obtained for all images in the 1097 frame PIV sequence (excluding the low-seeding density combustion product region), the remainder being removed and replaced with interpolated values. Every fourth image (500 $\mu$ s time delay) was cross-correlated for best accuracy at the relatively low velocities encountered at the stabilization point.

\section{F. Flame Propagation Velocity Measurement}

The 2-D components of the flame propagation velocitiy $V_{p}$, the flame velocity $V_{f}$, and the gas velocity $\mathrm{V}_{\mathrm{g}}$ in the CPIV image plane were obtained. $\mathrm{U}_{\mathrm{p}}, \mathrm{U}_{\mathrm{f}}$, and $\mathrm{U}_{\mathrm{g}}$ are the corresponding 2-D components in the direction normal to the thermal boundary surface at the stabilization point (parallel to the jet axis). Eqs. (2-4) below define $U_{p}, U_{f}$, and $U_{g}$ :

$$
\begin{aligned}
& \mathrm{U}_{\mathrm{p}}=\mathrm{V}_{\mathrm{p}} \bullet \mathrm{n} \\
& \mathrm{U}_{\mathrm{f}}=\mathrm{V}_{\mathrm{f}} \bullet \mathrm{n} \\
& \mathrm{U}_{\mathrm{g}}=\mathrm{V}_{\mathrm{g}} \bullet \mathrm{n}
\end{aligned}
$$

Substituting Eqs. (2-4) into Eq. (1) gives Eq. (5) below, which shows the relationship between $U_{p}, U_{f}$, and $U_{g}$ :

$$
\mathrm{U}_{\mathrm{p}}=\mathrm{U}_{\mathrm{f}}-\mathrm{U}_{\mathrm{g}}
$$

The flame velocity $U_{f}$ values were obtained from the stabilization point axial position time history. The axial and lateral position sequences were median and mean filtered to minimize the influence of random uncertainties. Filtering was not performed across discontinuities in the position sequence. The instantaneous value of $U_{f}$ is the first derivative of the axial position time history. A five-point central difference approximation of the first derivative was applied to obtain the $U_{f}$ values from the filtered axial position values. The stabilization point gas velocity $\mathrm{U}_{\mathrm{g}}$ values are the CPIV gas axial velocity values at the corresponding stabilization point position for each image in the sequence. The median and mean filtered gas velocities $\mathrm{U}_{\mathrm{g}}$ were subtracted from the stabilization point velocities $U_{f}$ to obtain the flame propagation 
velocity $U_{p}$ sequence.

\section{$\underline{\text { Results }}$}

\section{A. CPIV Velocity Field Images at 8000 Images/s}

A 1097 CPIV image sequence of the gas velocity field was obtained at an image acquisition rate of 8000 images/s. The time separation between sequential images is $125 \mu \mathrm{s}$ and the total time elapsed during the sequence is $137 \mathrm{~ms}$. Only the components of the gas velocities in the CPIV image plane are measured. Fig. 3a shows a sample CPIV image. The jet axis is just outside the field of view at the top of the image and the main jet flow is from left to right. The gas velocities are shown by vectors with a length proportional to the velocity magnitude. The flame boundary is shown by a thick line. The high-temperature flame product region is to the right of the thick line. The flame stabilization point is the farthest left (upstream) point on the boundary. Streamlines (thin lines) show the diverging flow pattern around the flame base. As expected, the highest velocities are nearest the jet axis and gradually decrease away from it. The velocity magnitude at the stabilization point is approximately $600 \mathrm{~mm} / \mathrm{s}$.

\section{B. Flame Propagation Velocity}

1097 measurements of $U_{p}, U_{f}$, and $U_{g}$ were obtained over a period of $137 \mathrm{~ms}$. Fig. 4 shows the measured values of $U_{f}$ and $U_{g}$ at the flame stabilization point. All velocities are normalized by the stoichiometric laminar flame speed $S_{L}$, which is $375 \mathrm{~mm} / \mathrm{s}$ for the methane/nitrogen fuel jet mixture. Note the positive correlation between these two data sets. Both follow the same trend and their maxima and minima occur together.

Fig. 5 shows the measured flame propagation velocity $\mathrm{U}_{\mathrm{p}}$, which is the difference between $\mathrm{U}_{\mathrm{f}}$ and $\mathrm{U}_{\mathrm{g}}$. The average values of $U_{p}, U_{f}$, and $U_{g}$ for this sequence are $0.19 \mathrm{~S}_{\mathrm{L}}, 0.62 \mathrm{~S}_{\mathrm{L}}$, and $0.81 \mathrm{~S}_{\mathrm{L}}$, respectively. The maximum and minimum values of $\mathrm{U}_{\mathrm{f}}$ are $2.6 \mathrm{~S}_{\mathrm{L}}$ and $-1.5 \mathrm{~S}_{\mathrm{L}}$. The maximum and minimum values of $U_{\mathrm{g}}$ are $2.3 \mathrm{~S}_{\mathrm{L}}$ and $-0.98 \mathrm{~S}_{\mathrm{L}}$. The positive correlation between the instantaneous values of $\mathrm{U}_{\mathrm{f}}$ and $\mathrm{U}_{\mathrm{g}}$ and the similar measured average and extreme values of $U_{f}$ and $U_{g}$ suggest that fluctuations in the flame position are influenced by fluctuations in the gas velocity field.

\section{Flame-Induced Flow Reversals}

Brief $\left(2-8 \mathrm{~ms}\right.$ ) periods of flow reversal (negative $U_{g}$ ) at the stabilization point are observed at times $t=24,63$, and $82 \mathrm{~ms}$, as shown in Fig. 4. Each occurrence of flow reversal is accompanied by negative flame velocities, inward gas velocities, and eddy passage just inward from the stabilization point. The maximum magnitude of the negative gas velocity $U_{g}$ in Fig. 4 is $0.98 \mathrm{~S}_{\mathrm{L}}$. Fig. 3 shows a CPIV image (a) with a close-up (b) of the reversal region immediately upstream of the stabilization point, at $\mathrm{t}=24 \mathrm{~ms}$. An isolated region of reverse flow is apparent just upstream of the flame stabilization point. The region of reverse flow is bordered on all sides by regions with positive gas velocities. Lateral velocity components within the reverse flow region are towards the jet centerline. The periods of reverse flow are accompanied by passage of turbulent eddies just inward of the flame stabilization point. Fig. 6 is a time history of the vorticity field including the flow reversal at $\mathrm{t}=24 \mathrm{~ms}$. Only every tenth image is shown for brevity. The boxed regions in Fig. 6 identify a pair of turbulent eddies (relatively small, isolated high vorticity regions) that move past and inward of the stabilization point. One of the eddies appears to cross the flame boundary between times $t=22.375 \mathrm{~ms}$ and $t=23.625 \mathrm{~ms}$. The eddies induce $a$ reduced, inward radial velocity component as they pass the stabilization point. The velocity induced by the turbulent eddies may combine with the velocity induced by the flame to create the flow reversal. The time history of the eddy motion shows that the eddies appear a significant distance upstream of the stabilization point, suggesting that they influence the flow field near the flame base rather than being merely created by it.

Negative values of the flame propagation velocity $U_{p}$ were measured at several times, as shown in Fig. 5. These periods are strongly correlated with positive $U_{\mathrm{f}}$. This correlation is reflected by the significant difference between the overall average $U_{\mathrm{f}}\left(0.62 \mathrm{~S}_{\mathrm{L}}\right)$ and the average $U_{f}$ conditioned on negative $U_{p}$ values (1.39 $S_{L}$ ). The negative $U_{p}$ values are believed to be caused by out-of-plane effects. Consider a case in which the reaction at the flame stabilization point is extinguished. The full 3-D flame propagation velocity $V_{p}$ is then zero, and any apparent motion of the boundary is due to convection. If the thermal boundary surface is perpendicular to the CPIV image plane and/or gas velocities $\mathrm{V}_{\mathrm{g}}$ in the CPIV image plane have no out-ofplane component, then the thermal boundary velocity $V_{f}$ is identical to the gas velocity $V_{g}$. However, a sloped thermal boundary surface convected by an outof-plane $V_{g}$ component can lead to an observed flame propagation velocity $U_{p}$ in the 2-D CPIV image plane, even if the full 3-D flame propagation velocity $V_{p}$ is zero. This observed velocity can be positive or negative, depending on the relationship between the signs of the out-of-plane thermal boundary slope and gas velocity. Fig. 7 illustrates the influence of out-ofplane thermal boundary slopes and gas velocities on the flame propagation velocity $U_{p}$ observed in the CPIV image plane.

\section{Flame Stabilization Point Motion}

The coordinates of the flame stabilization point are acquired at a high rate compared to the characteristic 
time scales of the flow, providing finely time-resolved observations of short-lived transient phenomena. A sequence of 549 discrete coordinate measurements is obtained at $250 \mu$ intervals spanning $137 \mathrm{~ms}$. Fig. 8 shows the axial and lateral position of the flame stabilization point relative to the lower-left corner of the field of view (55 mm axial distance from jet exit, 33 $\mathrm{mm}$ lateral distance from the jet axis).

A discontinuous jump in the stabilization point axial position is observed at $t=10.875 \mathrm{~ms}$ in Fig. 8a. This jump appears in the CPIV image sequence as an isolated pocket of products that suddenly appears upstream of the previous stabilization point and rapidly grows and connects with the downstream product zone. Fig. 9 is a sequence showing the appearance and growth of the isolated pocket from $t=10.875 \mathrm{~ms}$ to $t=13.625$ ms. It is speculated that appearance of the isolated pocket is due to out-of-plane propagation/convection of the flame thermal boundary into the CPIV image plane.

The lateral position of the stabilization point moves in a cyclic fashion. The lateral position moves progressively outward from the jet centerline until a new stabilization point appears much closer to the centerline, beginning a new cycle. Fig. $8 \mathrm{~b}$ shows several cycles of the lateral stabilization point motion.

\section{Conclusions}

1. A newly-developed high-speed Cinema PIV (CPIV) technique provides unique time-resolved images of the velocity field and flame boundary at $8000 \mathrm{images} / \mathrm{s}$ in a lifted turbulent jet diffusion flame.

2. Simultaneous measurement of the gas velocity field and thermal boundary velocity provides direct measurements of the flame propagation velocity at the stabilization point.

3. The average in-plane flame propagation velocity at the stabilization point is less than the laminar flame speed $S_{\mathrm{L}}$.

4. Time variations of the gas velocity and flame velocity are positively correlated, suggesting a strong interaction between the gas velocity field and the flame.

5. Brief periods of reverse flow are observed in an isolated region immediately upstream of the flame stabilization point. The maximum magnitude of the upstream gas velocity component is $0.98 \mathrm{~S}_{\mathrm{L}}$. Flow reversal is correlated in all observed cases with upstream flame motion, inward radial gas velocities, and eddy passage just inward of the stabilization point. The flow reversal may be caused by thermal expansion associated with the combustion process.

6. Rapid upstream jumps in the stabilization point axial position are occasionally observed. These events are thought to be the result of out-of-plane convection and/or propagation of the flame boundary.

7. Cyclic lateral motion of the stabilization point is observed. The period of the cycle is approximately $35 \mathrm{~ms}$.

\section{Acknowledgments}

This work was supported by NSF Grant CTS-9904198. We also acknowledge the cooperation between the Mechanical and Aerospace Engineering departments at the University of Michigan, which provided the combination of resources essential to the success of this project.

\section{References}

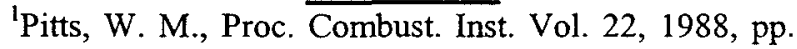
809-816

${ }^{2}$ Vanquickenborne, L. and van Tiggelen, A., Combustion and Flame, Vol. 10, 1966, pp. 59-69.

${ }^{3}$ Schefer, R. W., Namazian, M., Kelly, J., Proc. Combust. Inst. Vol. 22, 1988, pp. 833-842.

${ }^{4}$ Muñiz, L. and Mungal, M. G., Combustion and Flame, Vol. 111, pp 16-31.

${ }^{5}$ Watson, K. A., Lyons, K. M., Donbar, J. M., Carter, C. D., Combustion and Flame, Vol. 117, 1999, pp. 257-271.

${ }^{6}$ Hasselbrink, E. F. JR. and Mungal, M. G., Proc. Combust. Inst. Vol. 27, 1998, pp. 867-873.

${ }^{7}$ Brockhinke, A., Andresen, P., and Kohse-Höinghaus, K., Proc. Combust. Inst. Vol. 26, 1996, pp. 153-159.

${ }^{8}$ Tacke, M. M., Geyer, D., Hassel, E. P., Janicka, J., Proc. Combust. Inst. Vol. 27, 1998, pp. 1157-1165.

${ }^{9}$ Schefer, R. W., Namazian, M., Filtopoulos, E. E. J., and Kelly, J., Proc. Combust. Inst. Vol. 25, 1994, pp. 1223-1231. 


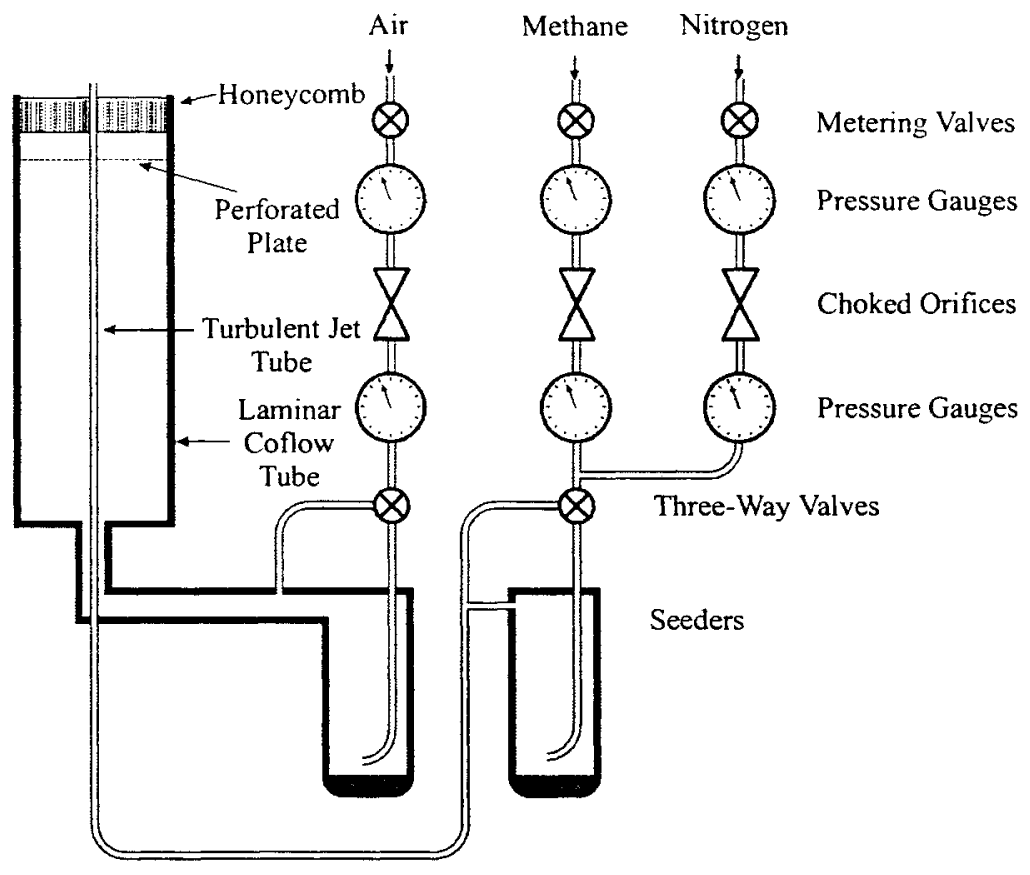

Fig. 1 Schematic of the lifted flame apparatus. Fuel jet inner diameter is 5.0 $\mathrm{mm}$; coflow air diameter is $170 \mathrm{~mm}$.

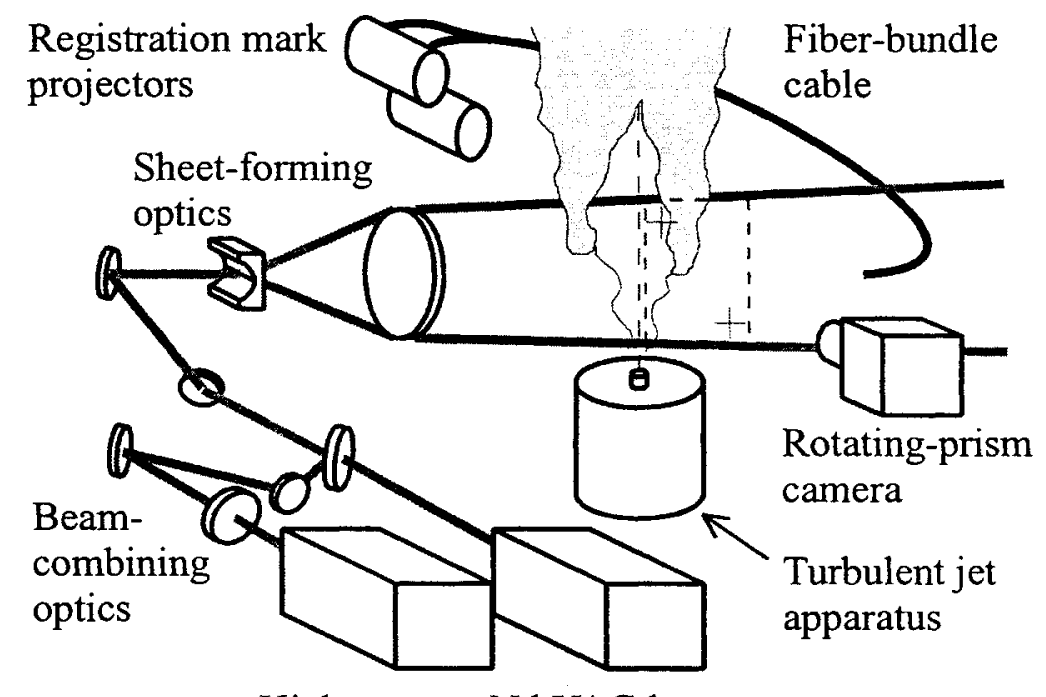

High rep-rate Nd:YAG lasers

Fig. 2 Schematic of the Cinema PIV diagnostics. 


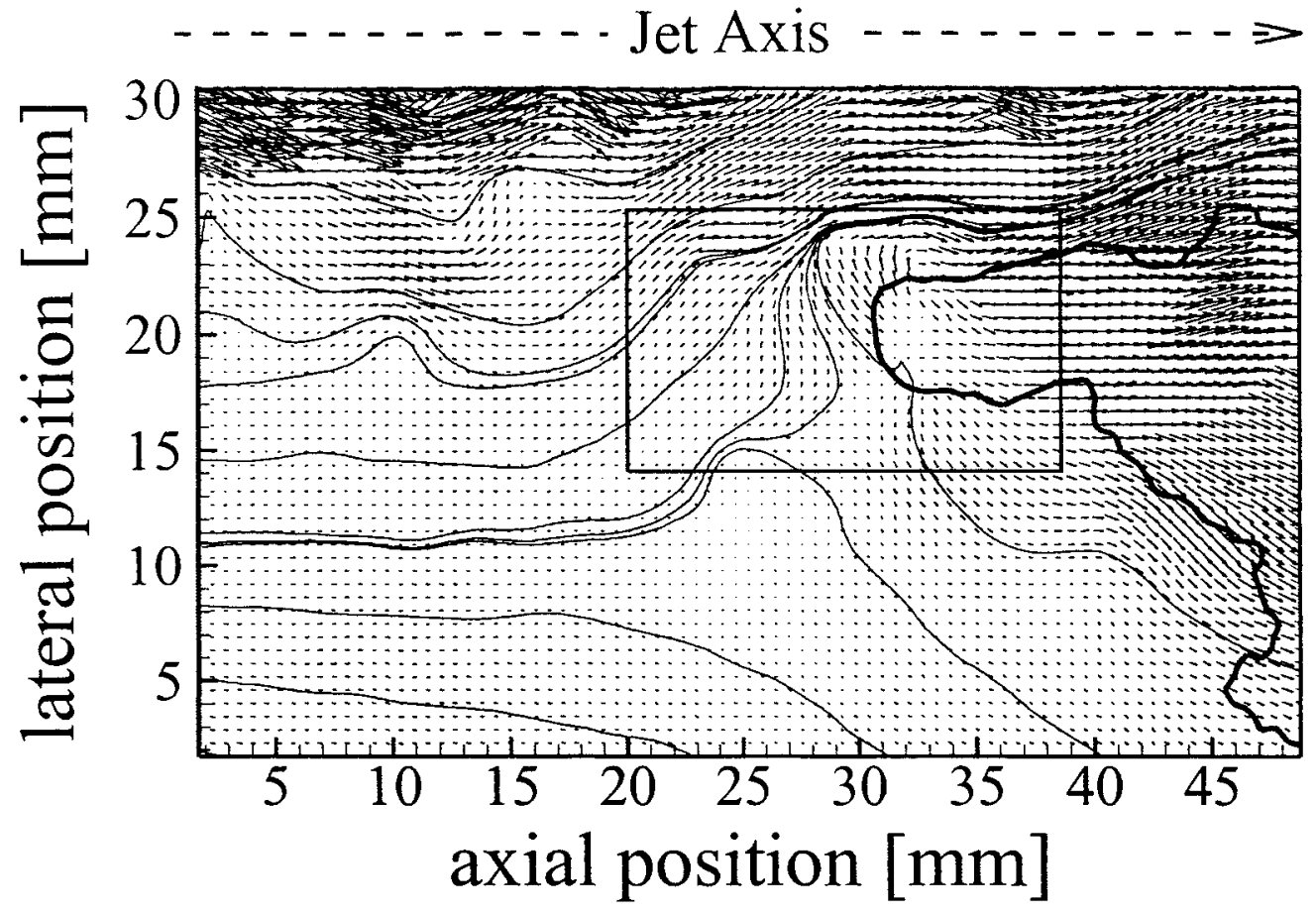

a)

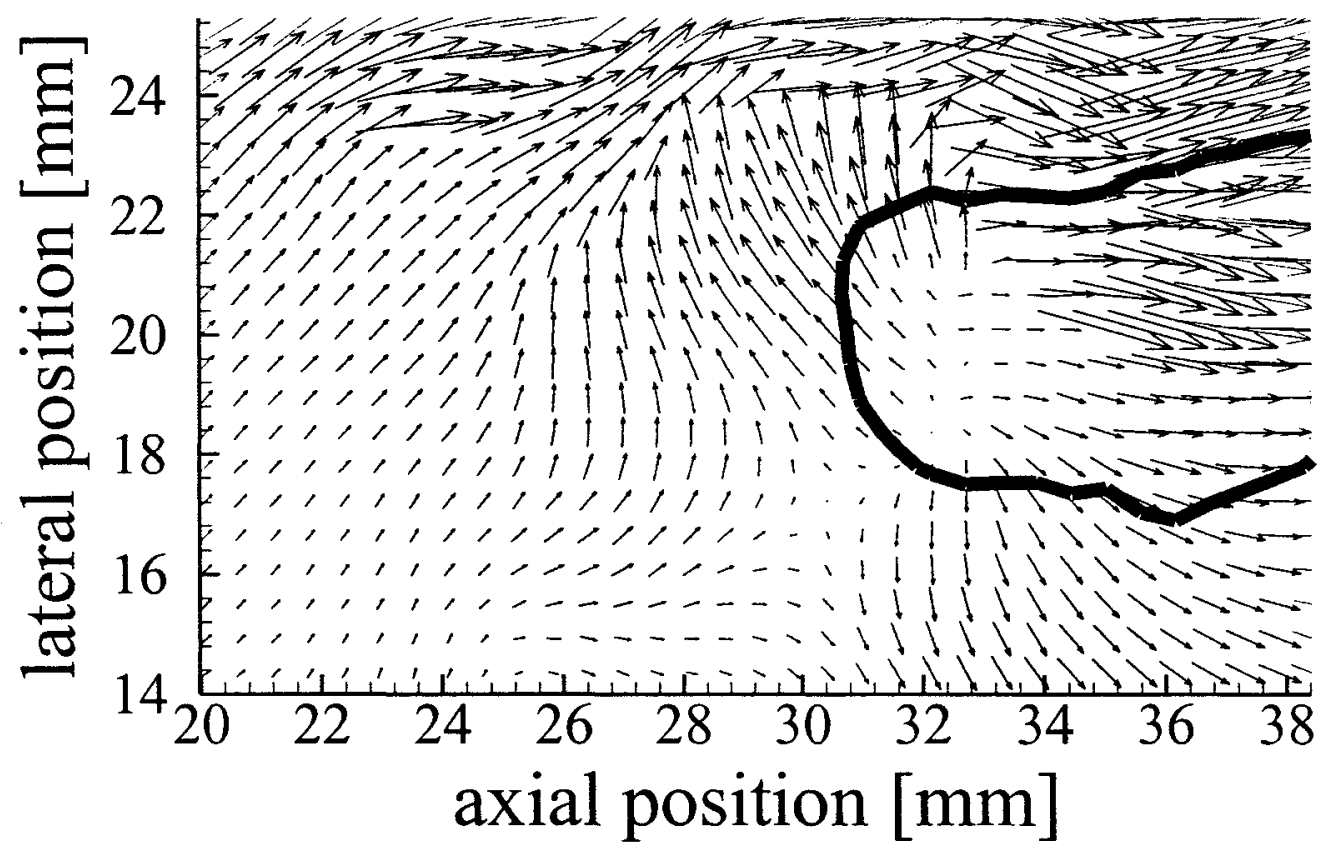

b)

Fig. 3 CPIV velocity field image showing gas velocities (vectors), the flame thermal boundary (thick line), and streamlines (thin lines). Entire field of view (a); close-up of boxed region (b). 


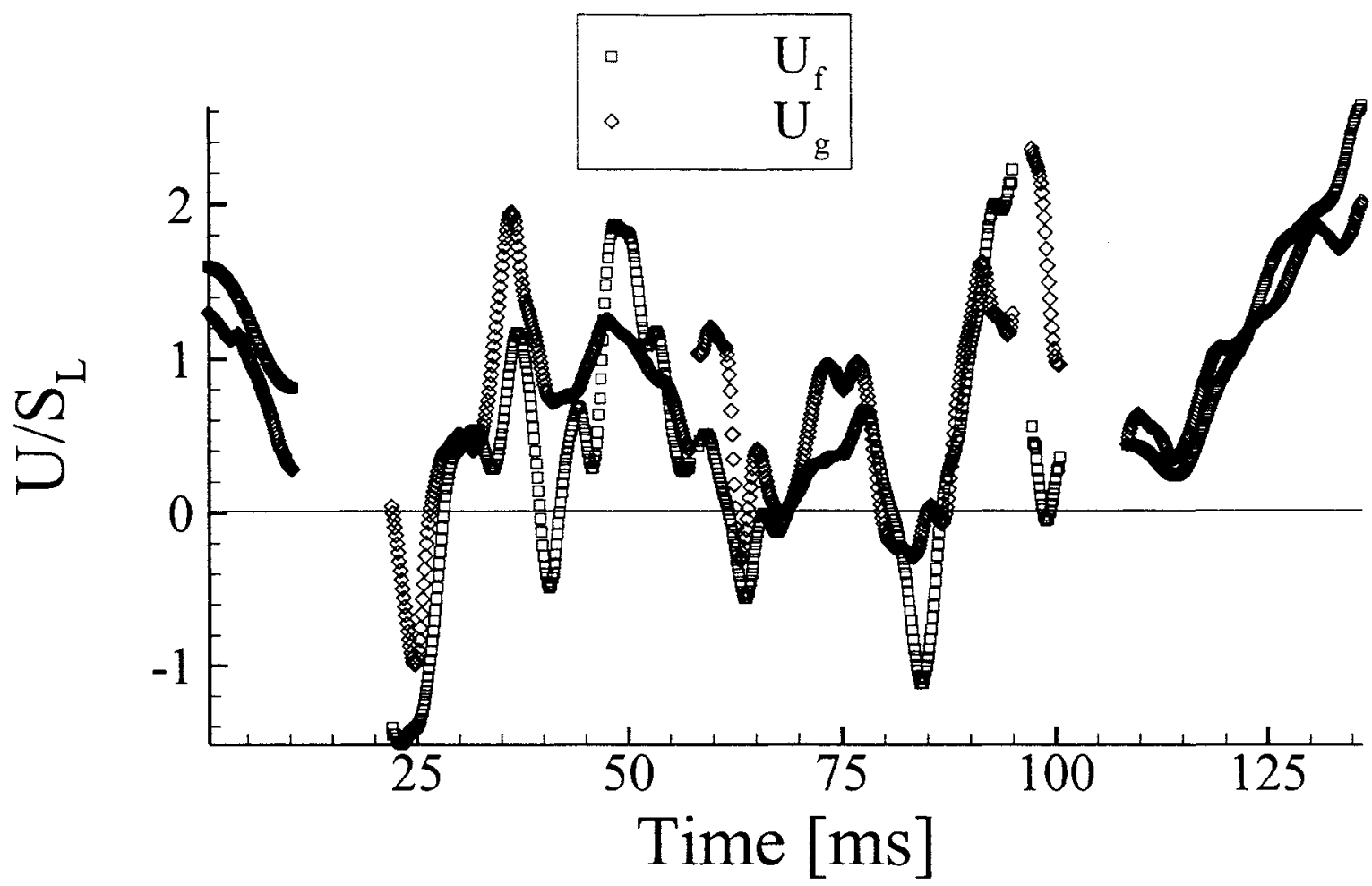

Fig. 4 Flame velocity $U_{\mathrm{f}}$ and gas velocity $\mathrm{U}_{\mathrm{g}}$ at the stabilization point.

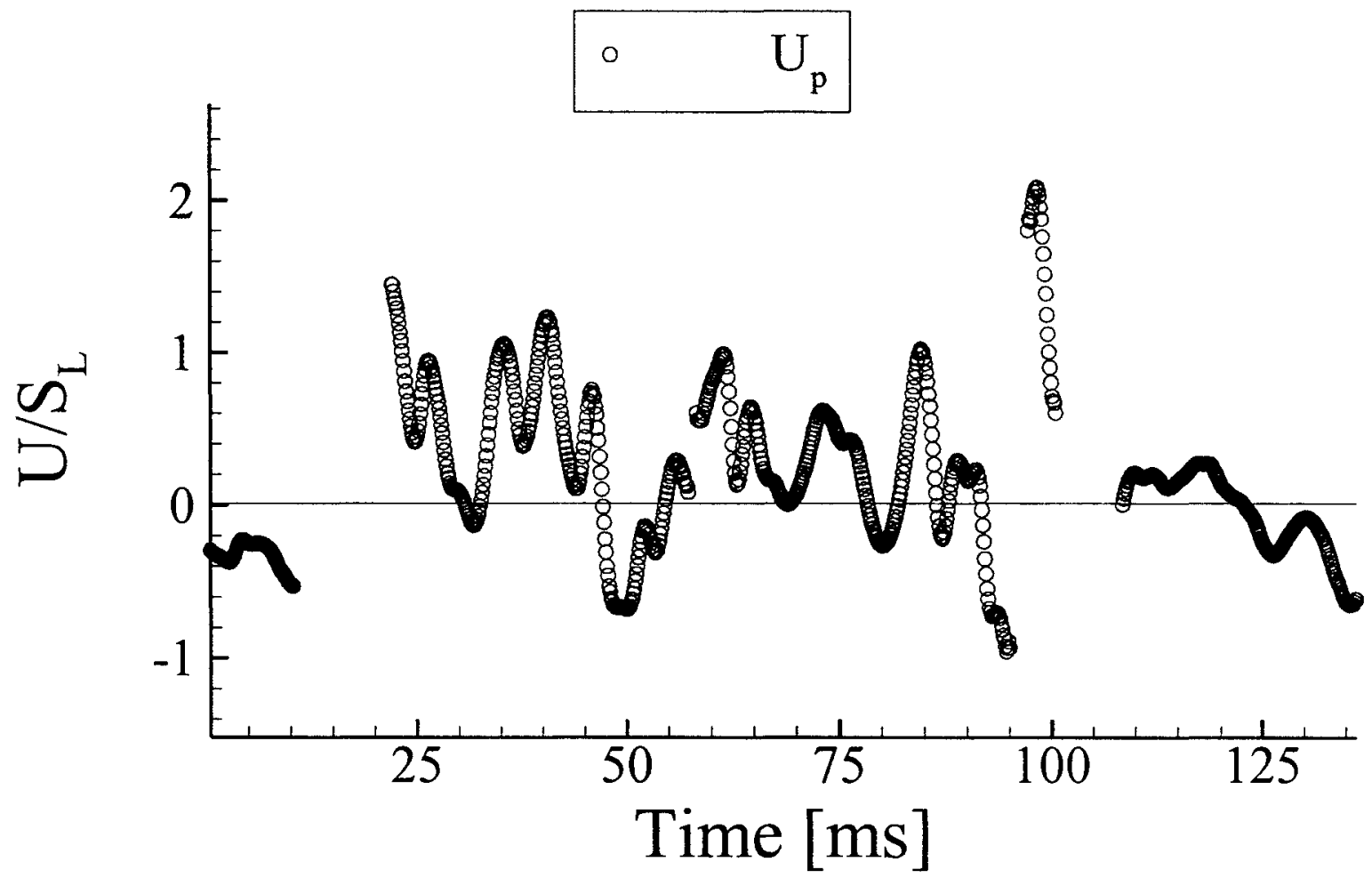

Fig. 5 Flame propagation velocity $\mathrm{U}_{\mathrm{p}}$ at the stabilization point. 

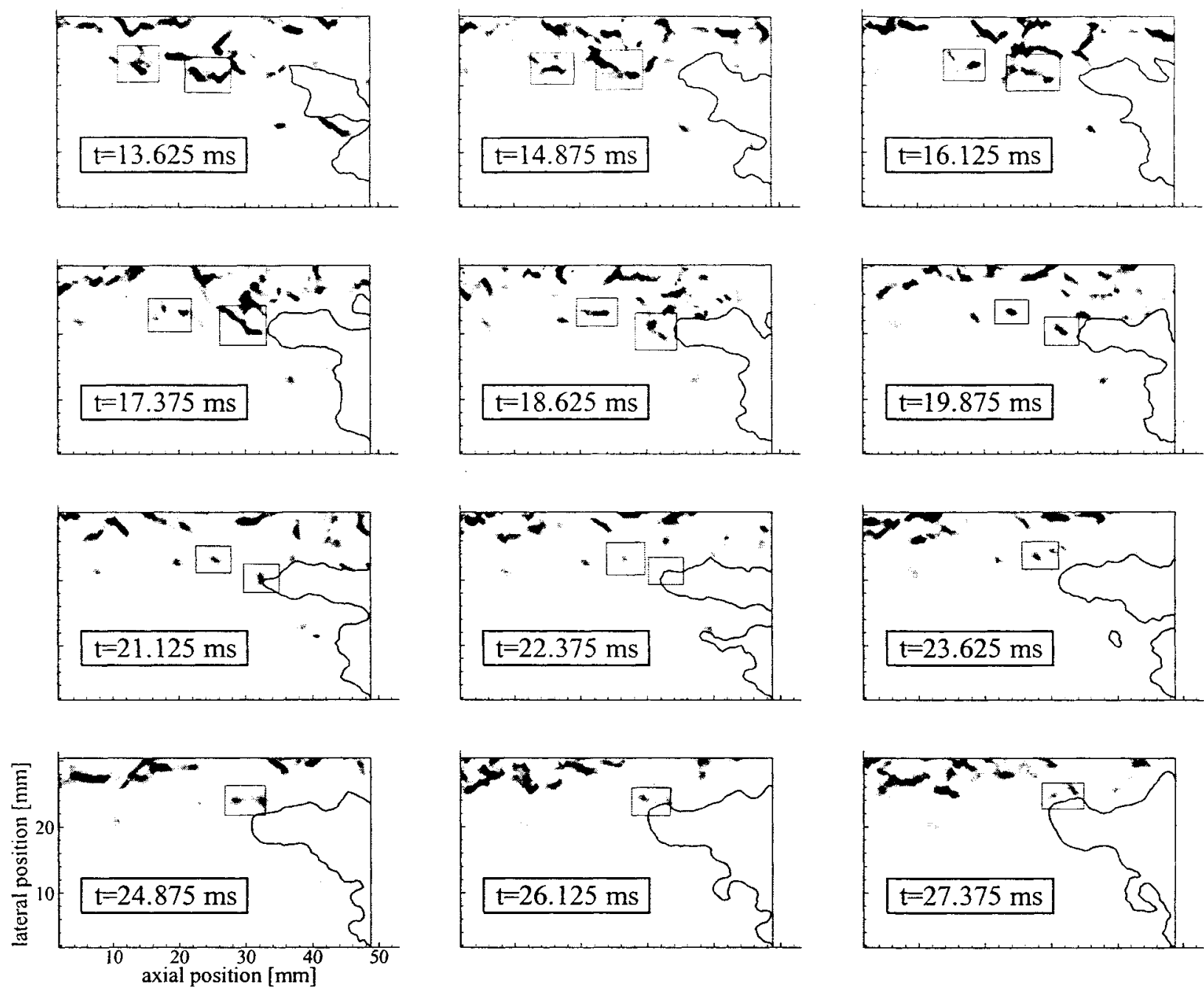

Fig. 6 Time sequence of flame boundary (thick line) and vorticity field (shading) showing trajectories of turbulent vortex structures. High vorticity (clockwise) regions are darkly shaded. Turbulent eddies which pass just inward of the flame stabilization point are boxed.
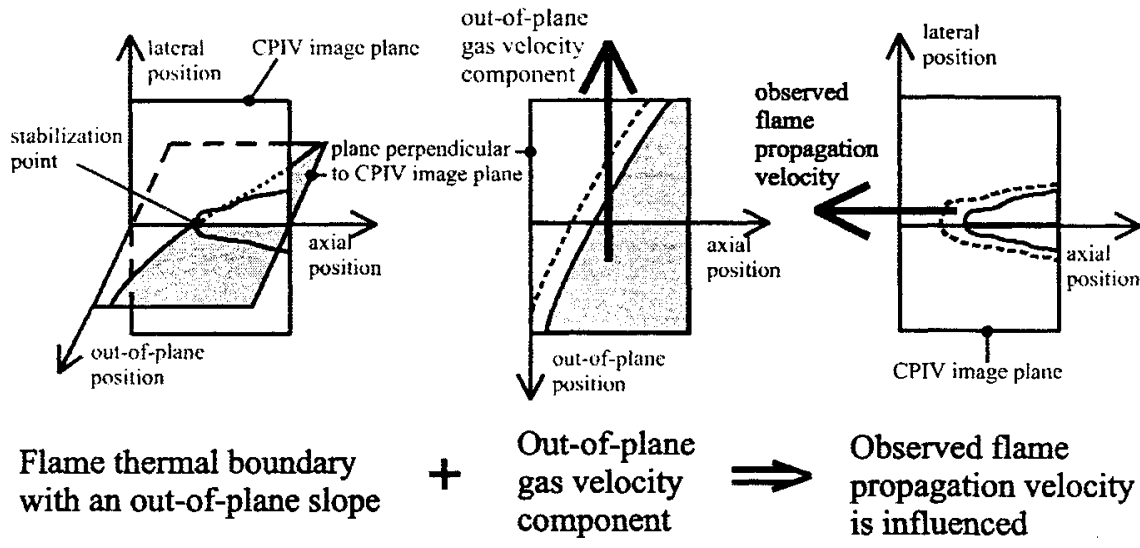

Fig. 7 The influence of out-of-plane flame boundary slopes and gas velocities on the flame propagation velocity. 


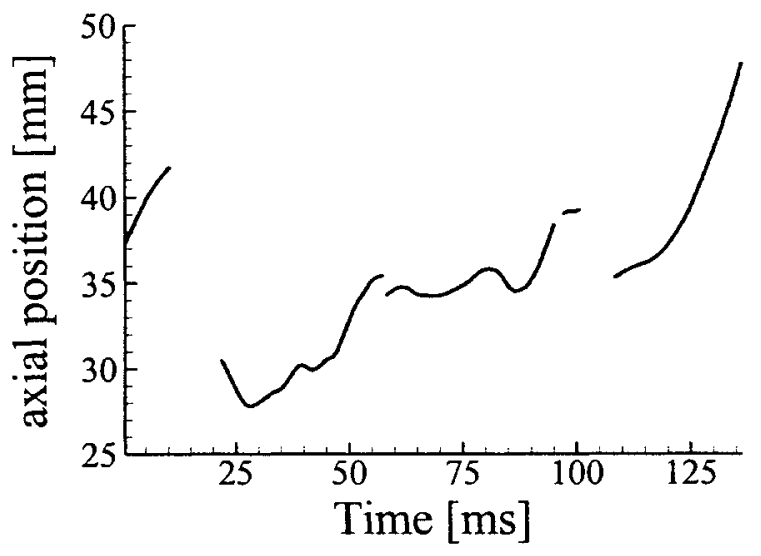

a)

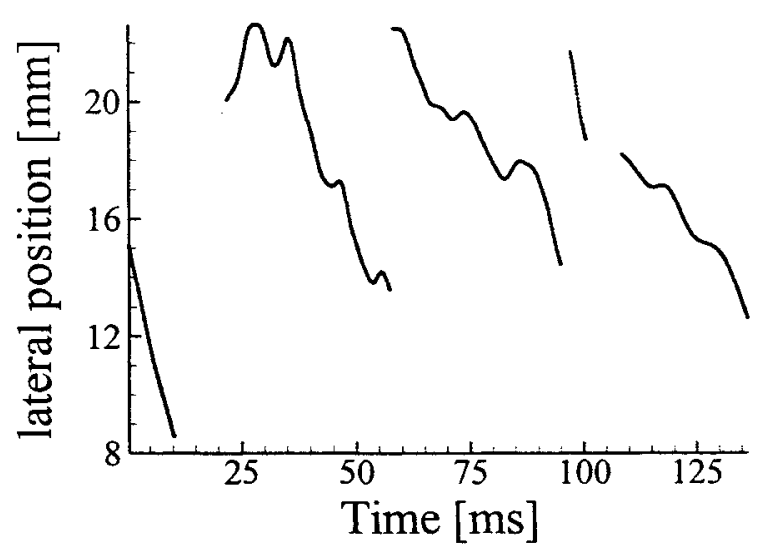

b)

Fig. 8 Position of the flame stabilization point. Axial position (a); lateral position (b).
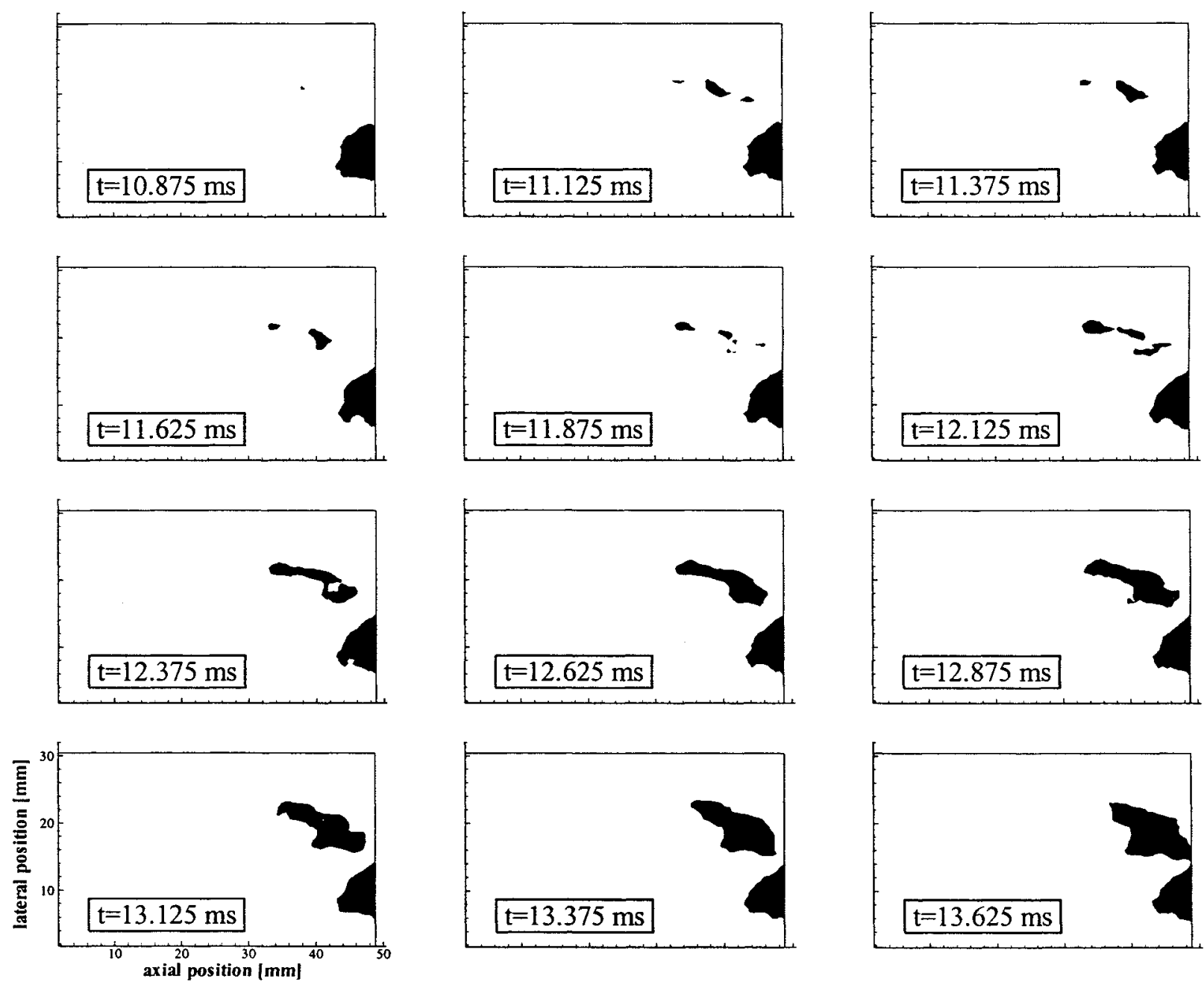

Fig. 9 Time sequence of flame boundary showing appearance and growth of an isolated pocket. 